\title{
THE BLOCKS OF THE PERIPLECTIC BRAUER ALGEBRA IN POSITIVE CHARACTERISTIC
}

\author{
SIGISWALD BARBIER, ANTON COX, AND MAUD DE VISSCHER
}

\begin{abstract}
We determine the blocks of the periplectic Brauer algebra over any field of odd positive characteristic.
\end{abstract}

\section{INTRODUCTION}

The periplectic Brauer algebra belongs to a class of algebras which can be represented using diagrams. Other examples of diagram algebras include the symmetric group algebra, the Hecke algebra, the Temperley-Lieb algebra and the Brauer algebra.

The periplectic Brauer algebra was first introduced by Moon [Moo03] to study the periplectic Lie superalgebra. This is similar to the way Schur-Weyl duality is used to relate representation theory of the symmetric group to representation theory of the general linear group and representation theory of the Brauer algebra to the orthogonal and symplectic Lie algebra or to the encompassing orthosymplectic Lie superalgebra [BSR98, ES16, LZ17].

The periplectic Brauer algebra $A_{n}$ is closely related to the Brauer algebra $B_{n}(\delta)$ for $\delta=0$, [Se14, KT17]. For example, they can both be represented using the same Brauer diagrams and with multiplication only differing up to a minus sign. So it should come as no surprise that certain aspects of the representation theory of the periplectic Brauer algebra resembles the representation theory of the Brauer algebra. For instance, their simple modules can be labelled by the same partitions. However, there are also striking differences. While the Brauer algebra is cellular, this is no longer the case for the periplectic Brauer algebra. Also the description of the blocks in characteristic zero is quite different, and we will show this is still the case in positive characteristic.

The representation theory of the Brauer algebra has already been developed for a long time [KX01, CDM09a, CDM09b, Kin14, Ma15]. In contrast the representation theory of the periplectic Brauer algebra remained unstudied until quite recently. In particular the simple modules have been classified for arbitrary characteristic [KT17] and for characteristic zero (or large characteristic) a classification of the blocks [Cou18] and a complete description of the decomposition multiplicities [CE18] have been obtained.

Calculating the decomposition multiplicities of the (periplectic) Brauer algebra in positive characteristic is an important open problem. Since this is related to the long-standing open problem of the decomposition multiplicities of the symmetric group, a solution to this problem seems currently not within reach. Instead, as a first step, we obtain in this paper a complete classification of the blocks of the periplectic Brauer algebra in all (odd) positive characteristic.

There are a number of technical challenges that have to be addressed along the way. As the periplectic Brauer algebras are not cellular, we need to work in the setting of standardly based 
algebras, where a more limited set of tools are at our disposal. There is also a much more delicate interplay with the representation theory of the symmetric group: for example we develop a version of BGG reciprocity for these algebras, but with a twist arising from the (highly nontrivial) Mullineux map on representations of the symmetric group (Theorem 7.3).

This leads to a very different classification of blocks from the classical Brauer case in Theorem 8.11. For example we will see that if $n$ is not too small compared to the characteristic then there is only one block (and will give a complete classification in terms of certain staircase partitions in the general case). Note that this result is better than in the Brauer algebra case where only the limiting blocks are known in positive characteristic [Kin14].

This paper is structured as follows. In Section 2 we introduce the periplectic Brauer algebra. As mentioned, the periplectic Brauer algebra fails to be cellular, but it still satisfies the weaker notion of a standardly based algebra. In Section 3, we recall the definitions and properties of standardly based algebras needed in this paper. We also review the relevant partition combinatorics and representation theory of the symmetric group algebra in Section 4. In Section 5 we combine all this information to obtain a standard basis of the periplectic Brauer algebra and an explicit description of the standard modules. Using localisation and globalisation functors in Section 6, we obtain a full embedding of the module category $A_{n}$-mod into $A_{n+2}$-mod. This can then be used for induction arguments. In particular, using the results of Sections 5 and 6 we prove a BGG-reciprocity for the periplectic Brauer algebra in arbitrary characteristic in Section 7. Finally, we obtain a complete description of the blocks of the periplectic Brauer algebra in any characteristic $p \neq 2$ in Section 8.

We will wish to compare the representation theory of our algebras in characteristic $p$ to characteristic zero, and so will use the following conventions throughout this paper. We will consider a $p$-modular system $(\mathbb{K}, R, \mathbb{k})$, which means that $R$ is a discrete valuation ring, $\mathbb{K}$ is the associated field of fractions, which will be of characteristic zero, and $\mathbb{k}$ is the quotient of $R$ by its maximal ideal, and is a field of characteristic $p>0$. If we wish to consider an arbitrary field we will denote it by $k$.

Throughout the paper, unless otherwise stated, all the modules will be finite dimensional left modules.

\section{The Periplectic Brauer Algebra}

The periplectic Brauer algebra was first introduced by Moon [Moo03] in terms of generators and relations. However we will use the diagrammatic definition due to Kujawa and Tharp [KT17]. This is very similar to the definition of the Brauer algebra in terms of Brauer diagrams, but with a deformed version of multiplication as defined below. The periplectic Brauer algebra also appears in [BDEal18] where it is defined as an endomorphism algebra in the Brauer supercategory (a monoidal supercategory generated by a single object).

An $(r, s)$-Brauer diagram is a partition of $r+s$ nodes into (unordered) pairs. It can be represented pictorially by $r$ nodes on a (northern) horizontal line and $s$ nodes on a second (southern) horizontal line below the first one, with an edge between two nodes if they belong to the same pair. An edge which connects two nodes on the northern line is called a cup, an edge which connects two nodes on the southern line is called a cap, and an edge which connects a node on the northern line with a node on the southern line is called a propagating line. An example of a $(6,8)$-Brauer diagram is given in Figure 1. 


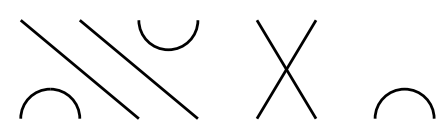

FiguRE 1. An example of a $(6,8)$-Brauer diagram

To compose an $(r, s)$-Brauer diagram $d_{1}$ with an $(s, t)$-Brauer diagram $d_{2}$ we will need the notion of a marked Brauer diagram. We decorate Brauer diagrams with certain markings as follows, and will choose a preferred decoration to be the standard marking.

To produce a marked Brauer diagram we choose a marking for each cup and cap, and a linear order on them, as follows. Each cup is marked with a diamond $\diamond$, and each cap with either a left arrow $\triangleleft$ or a right arrow $\triangleright$. Given an arbitrary linear ordering on the caps and cups, we depict this by placing the markings on different latitudes between the northern and southern horizontal lines, such that a cup or cap which is larger than another cup or cap has the more northerly latitute of the two. (In order to do this we may need to deform our Brauer diagram, but as these are only considered up to isotopy this does not affect the definition.) We say that two markings are adjacent if there is no other marking between them in this order.

We can now define a standard marking for each Brauer diagram. First we choose to mark all caps with right arrows. For our ordering, we set all the cups to be larger than all the caps. Then we say that one cup is larger than another cup if its leftmost node is to the left of the leftmost node of the other cup. Finally we say that a cap is larger than another cap if its leftmost node is to the right of the leftmost node of the other cap.

Two examples of Brauer diagrams with markings are given in Figure 2. The righthand diagram has the standard marking.
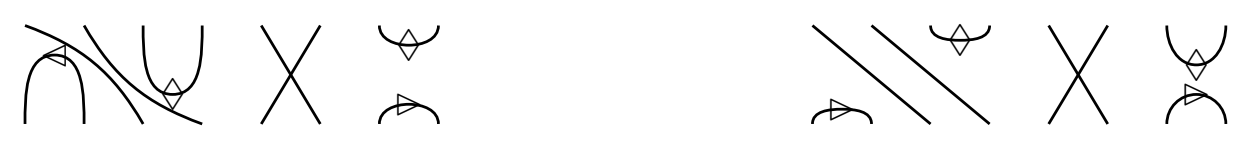

FiguRE 2. A marked and a standardly marked Brauer diagram

Using these markings we define the composition $d_{1} d_{2}$ of Brauer diagrams $d_{1}$ and $d_{2}$ as follows. If the number of nodes on the southern line of $d_{1}$ is different from the number of nodes on the northern line of $d_{2}$, we set $d_{1} d_{2}$ equal to zero. Otherwise we concatenate the two Brauer diagrams by identifying the northern horizontal line of $d_{2}$ with the southern line of $d_{1}$ to obtain a new diagram $d_{1} \star d_{2}$. If this new diagram contains closed loops, we set $d_{1} d_{2}$ zero. Otherwise $d_{1} \star d_{2}$ is again a Brauer diagram and we set

$$
d_{1} d_{2}=(-1)^{\gamma\left(d_{1}, d_{2}\right)} d_{1} \star d_{2}
$$

where $\gamma\left(d_{1}, d_{2}\right)$ is defined as follows.

First equip $d_{1}$ and $d_{2}$ with their standard marking. This will give us a decoration on $d_{1} \star d_{2}$, possibly with more than one marking on the same edge. We can make this into a standard marking by combinations of the following operations:

(i) if two markings are adjacent, we switch their order,

(ii) if a cap has a left arrow we switch it to a right arrow, and 
(iii) if an arrow and a diamond are adjacent and on the same edge, we remove both markings.

Then $\gamma\left(d_{1}, d_{2}\right)$ counts the number of switching operations of type (i) and (ii), and the number of cancelling operations of type (iii) where the arrow points away from the diamond which are needed to obtain a standard marking on $d_{1} \star d_{2}$. Of course there may be many different ways to obtain the standard marking, but it is shown in [KT17] that $(-1)^{\gamma\left(d_{1}, d_{2}\right)}$ is independent of the chosen operations.

Definition 2.1. Let $k$ be a field. The periplectic Brauer algebra $A_{n}$ is the $k$-algebra with basis given by $(n, n)$-Brauer diagrams, and multiplication given by linear extension of the composition of Brauer diagrams defined in (1).

Remark 2.2. Note that in [KT17] they define $A_{n}$, as a vector space, to be the span of all marked $(n, n)$-Brauer diagrams subject to the switching relations (i) and (ii) (with signs) given above. The multiplication on $A_{n}$ is then defined by concatenation of marked diagrams and relation (iii) (with signs) given above. Using their definition, it is clear that the multiplication in $A_{n}$ does not depend on the choice of linear order used to define the standardly marked Brauer diagrams. They then prove that the set of standardly based marked diagrams forms a basis for $A_{n}$.

We may consider the category whose objects are natural numbers and where morphisms between $r$ and $s$ are given by $(r, s)$-Brauer diagrams. This can be given the structure of a (strict) monoidal supercategory in the sense of [BE17] by defining the tensor product of an $(r, s)$-Brauer diagram with an $\left(r^{\prime}, s^{\prime}\right)$-Brauer diagram as follows. We concatenate horizontally the first diagram with $r^{\prime}$ (non-crossing) propagating lines on the right and concatenate the second diagram with $s$ (non-crossing) propagating lines on the left. Then we take the composition defined in (1) of the $\left(r+r^{\prime}, s+r^{\prime}\right)$ and $\left(s+r^{\prime}, s+s^{\prime}\right)$ diagrams obtained in this way. This gives us a $\left(r+r^{\prime}, s+s^{\prime}\right)$ Brauer diagram with a possible minus sign. Then the monoidal supercategory is generated by the elements $I, X, \cap$ and $\cup$ where $I$ is the unique $(1,1)$-Brauer diagram, $\cap$ and $\cup$ are the unique $(0,2)$ - and $(2,0)$-Brauer diagrams, and $X$ is the $(2,2)$-Brauer diagram with two propagating lines that cross precisely once [KT17, Theorem 3.2.1].

Definition 2.3. We define a contravariant autoequivalence $\phi$ on this category as follows. The map $\phi$ fixes objects, and we set

$$
\phi(I)=I, \quad, \phi(X)=-X, \quad \phi(\cap)=\cup, \quad \phi(\cup)=-\cap
$$

and require $\phi(a b)=\phi(b) \phi(a)$ and $\phi(a \otimes b)=\phi(a) \otimes \phi(b)$. One can check that $\phi$ is well-defined and induces an algebra anti-automorphism $\phi$ on $A_{n}$, see [Cou18, Section 2.1.6].

The symmetric group algebra $\mathrm{H}_{n}=k \mathfrak{S}_{n}$ appears as the subalgebra of $A_{n}$ spanned by all diagrams with no caps (or cups). It also appears as the quotient of $A_{n}$ by the ideal generated by all diagrams containing at least one cap (or cup). It is easy to see that the anti-automorphism $\phi$ induces an anti-automorphism $\phi$ on this subalgebra or quotient given by

$$
\phi(w)=(-1)^{\ell(w)} w^{-1}
$$

for all $w \in \mathfrak{S}_{n}$.

The study of the representation theory of the Brauer algebras in [CDM09a, CDM09b] was based on the fact that these algebras were cellular (and frequently even quasihereditary). Unfortunately there is no obvious cellular structure in the marked Brauer case. However in [Cou18, Theorem 4.1.2], it is shown that $A_{n}$ is a standardly based algebra. This will be the general 
framework in which we need to work, and so we now recall the definition and some of the properties of such classes of algebras.

\section{StandARDLY BASED ALGEBRA}

We recall the notion of standardly based algebras introduced in [DR98]. In this section we work over an arbitrary field $k$. Let $(\Lambda, \geq)$ be a poset, $A$ be a finite dimensional $k$-algebra and $\mathcal{B}$ be a basis for $A$. We say that $(A, \mathcal{B})$ is a based algebra if we can write $\mathcal{B}$ as a disjoint union of subsets $\mathcal{B}^{\lambda}$ for $\lambda \in \Lambda$ such that for all $a \in A$ and $b \in \mathcal{B}^{\lambda}$ we have that $a b$ and $b a$ can be written as linear combinations of basis elements $c \in \mathcal{B}^{\mu}$ with $\mu \geq \lambda$. This allows us to define two-sided ideals of $A$ for each $\lambda \in \Lambda$, namely $A^{\geq \lambda}$ spanned by $\cup_{\mu \geq \lambda} \mathcal{B}^{\mu}$ and $A^{>\lambda}$ spanned by $\cup_{\mu>\lambda} \mathcal{B}^{\mu}$. We define $A^{\lambda}$ to be the $(A, A)$-bimodule $A^{\geq \lambda} / A^{>\lambda}$. We abuse notation and view $A^{\lambda}$ as the $k$-span of $\mathcal{B}^{\lambda}$. If we assume further that for each $\lambda \in \Lambda$ we have indexing sets $I(\lambda)$ and $J(\lambda)$ such that

$$
\mathcal{B}^{\lambda}=\left\{a_{i j}^{\lambda} \mid(i, j) \in I(\lambda) \times J(\lambda)\right\}
$$

and for each $a \in A$ and $a_{i j}^{\lambda}$ we have

$$
\begin{aligned}
& a a_{i j}^{\lambda}=\sum_{i^{\prime} \in I(\lambda)} f_{i^{\prime}, \lambda}(a, i) a_{i^{\prime} j}^{\lambda} \bmod A^{>\lambda} \text { and } \\
& a_{i j}^{\lambda} a=\sum_{j^{\prime} \in J(\lambda)} f_{\lambda, j^{\prime}}(j, a) a_{i j^{\prime}}^{\lambda} \bmod A^{>\lambda},
\end{aligned}
$$

then we say that $(A, \mathcal{B})$ is a standardly based algebra.

Note that if, in addition, we have an algebra anti-involution $\psi$ such that $\psi\left(a_{i j}^{\lambda}\right)=a_{j i}^{\lambda}$ then $(A, \mathcal{B})$ is a cellular algebra as defined in [GL96].

Now for each $\left(i_{0}, j_{0}\right) \in I(\lambda) \times J(\lambda)$ we can define the left $A$-module $\Delta\left(\lambda, j_{0}\right)$ (resp. the right $A$-module $\left.\Delta\left(i_{0}, \lambda\right)\right)$ to be the $k$-span of $\left\{a_{i j_{0}}^{\lambda} \mid i \in I(\lambda)\right\}$ (respectively of $\left\{a_{i_{0} j}^{\lambda} \mid j \in J(\lambda)\right\}$ ). As these modules are clearly independent of the choice of $\left(i_{0}, j_{0}\right)$ we write $\Delta(\lambda)=\Delta\left(\lambda, j_{0}\right)$ and $\Delta^{\mathrm{op}}(\lambda)=\Delta\left(i_{0}, \lambda\right)$ and call them the left, respectively right, standard modules for $A$. By definition, we have an isomorphism of $(A, A)$-bimodules $A^{\lambda} \cong \Delta(\lambda) \otimes_{k} \Delta^{\mathrm{op}}(\lambda)$.

It is shown in [DR98, 2.4] that there is a subset $\Lambda^{\prime} \subseteq \Lambda$ (defined in terms of a bilinear form on standard modules) such that for all $\lambda \in \Lambda^{\prime}$ we have that $L(\lambda):=\Delta(\lambda) / \operatorname{rad} \Delta(\lambda)$ is simple and moreover $\left\{L(\lambda) \mid \lambda \in \Lambda^{\prime}\right\}$ forms a complete set of pairwise non-isomorphic simple (left) $A$-modules. For each $\lambda \in \Lambda^{\prime}$ we denote by $P(\lambda)$ the projective cover of $L(\lambda)$.

Proposition 3.1. [DR98, (2.4.1),(2.4.4)] Let $\lambda \in \Lambda^{\prime}$ and $\mu \in \Lambda$.

(1) The composition multiplicity $[\Delta(\mu): L(\lambda)]$ satisfies

$$
[\Delta(\mu): L(\lambda)] \neq 0 \text { implies that } \lambda \leq \mu
$$

and $[\Delta(\lambda): L(\lambda)]=1$.

(2) The projective indecomposable module $P(\lambda)$ has a filtration by standard modules. If we denote by $(P(\lambda): \Delta(\mu))$ the number of sections isomorphic to $\Delta(\mu)$ in this filtration then we have

In particular we have

$$
(P(\lambda): \Delta(\mu))=\operatorname{dim}\left(\Delta^{\mathrm{op}}(\mu) \otimes_{A} P(\lambda)\right) .
$$

$$
(P(\lambda): \Delta(\mu)) \neq 0 \text { implies that } \mu \geq \lambda
$$

and $(P(\lambda): \Delta(\lambda))=1$. 
For $\lambda, \mu \in \Lambda^{\prime}$ we say that the two simple $A$-modules $L(\lambda)$ and $L(\mu)$ belong to the same block if there is a sequence $\lambda=\lambda_{1}, \lambda_{2}, \ldots, \lambda_{t}=\mu$ in $\Lambda^{\prime}$ and a sequence of indecomposable $A$-modules $M_{1}, M_{2}, \ldots M_{t-1}$ such that for each $1 \leq i \leq t-1$ we have that $L\left(\lambda_{i}\right)$ and $L\left(\lambda_{i+1}\right)$ appear as composition factors of $M_{i}$. This gives an equivalence relation on $\Lambda^{\prime}$ where each equivalence class corresponds to a block of $A$. Thus we will abuse notation and refer to elements $\lambda$ and $\mu$ of $\Lambda^{\prime}$ as being in the same block if $L(\lambda)$ and $L(\mu)$ lie in the same block for $A$.

Corollary 3.2. Every standard module occurs in the filtration of some projective indecomposable module, and hence has all composition factors belonging to a single block.

Proof. Let $\Delta(\mu)$ be a standard module for $A$. By Proposition 3.1(2) it is enough to show that

$$
\operatorname{dim}\left(\Delta^{\mathrm{op}}(\mu) \otimes_{A} P(\lambda)\right) \neq 0
$$

for some $\lambda \in \Lambda^{\prime}$. But $A \cong \bigoplus_{\lambda \in \Lambda^{\prime}} P(\lambda)^{a_{\lambda}}$ for some $a_{\lambda}>0$ and

$$
\operatorname{dim}\left(\Delta(\mu)^{\mathrm{op}} \otimes_{A} A\right)=\operatorname{dim}\left(\Delta(\mu)^{\mathrm{op}}\right) \neq 0
$$

and so there must exist some $\lambda$ such that (2) holds.

By Corollary 3.2 it makes sense to talk about the block of a standard module and hence to extend the block relation from $\Lambda^{\prime}$ to the whole of $\Lambda$.

\section{Partition COMbinatorics AND RePREsentations of the Symmetric Group}

Partition combinatorics. We briefly review the basic properties of partitions and Young digrams that we will need; further details can be found in [JK81]. Given $n \in \mathbb{N}$, a partition $\lambda$ of $n$ is an element $\lambda=\left(\lambda_{1}, \lambda_{2}, \ldots, \lambda_{k}\right)$ such that $\lambda_{i} \in \mathbb{N}$ for all $1 \leq i \leq k$, with $\lambda_{1} \geq \lambda_{2} \geq \cdots \geq \lambda_{k}$ and $|\lambda|:=\lambda_{1}+\lambda_{2}+\ldots \lambda_{k}=n$. If $\lambda$ is a partition of $n$ we write $\lambda \vdash n$.

We define a partial order $\unlhd$ on the set of all partitions (of any $n$ ) as follows. For two partitions $\lambda$ and $\mu$ we set $\lambda \unlhd \mu$ if and only if either $|\lambda|>|\mu|$ or $|\lambda|=|\mu|$ and $\sum_{i=1}^{j} \lambda_{i} \leq \sum_{i=1}^{j} \mu_{i}$ for all $j \geq 1$. (If $|\lambda|=|\mu|$ this is just the usual dominance order.)

We will often identify a partition $\lambda$ with its Young diagram. This is a collection of $n$ boxes ordered in left-justified rows such that the $i$ th row from the top contains $\lambda_{i}$ boxes. For example the Young diagram corresponding to $\lambda=(4,4,2,1)$ is illlustrated in Figure 3(a). The transpose $\lambda^{T}$ of a partition $\lambda$ is the partition whose corresponding Young diagram has as rows the columns of the Young diagram of $\lambda$. For example, the transpose of $\lambda=(4,4,2,1)$ is $\lambda^{T}=(4,3,2,2)$.

A hook of a Young diagram corresponding to a given box consists of that box and all boxes below it in the same column and all boxes to the right of it in the same row. We obtain the corresponding rim hook by pushing all boxes down and to the right until they get to the edge of the Young diagram. For example, the hook corresponding to the box $(1,2)$ of $\lambda=(4,4,2,1)$ is shown in Figure 3(b), and the corresponding rim hook is shown in Figure 3(c).

(a)

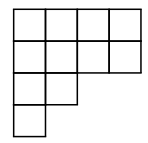

(b)

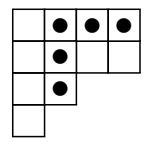

(c)

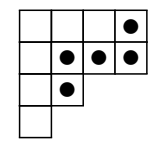

FIgURE 3. A Young diagram, the hook corresponding to $(1,2)$, and the associated rim hook. 
We say that a partition $\lambda$ is an $r$-staircase if

$$
\lambda=(r, r-1, \ldots, 1) .
$$

For example, if we remove the box $(2,4)$ from the partition in Figure 3(a) then we are left with a 4-staircase. Note that the maximal rim hook for an $r$-staircase has length $2 r-1$.

Fix a prime number $p$. A (rim) $p$-hook is a (rim) hook consisting of $p$ boxes. The $p$-core of a given partition $\lambda$ is the partition we obtain by successively removing rim $p$-hooks until this is no longer possible. The $p$-core is independent of the order in which one removes these $p$-hooks. Note that 2-cores are given by $r$-staircases for $r \geq 0$.

We say that $\lambda$ is p-restricted if $\lambda_{i}-\lambda_{i+1}<p$ for all $i$ and $p$-regular if there is no $i$ such that $\lambda_{i}=\lambda_{i+1}=\cdots=\lambda_{i+p-1}$ (that is if $\lambda^{T}$ is $p$-restricted). We will also say that any partition is 0 -regular and 0-restricted.

To each box $b$ in a Young diagram we associate a corresponding content by setting $\operatorname{con}(b)=j-i$ where $b$ is in row $i$ and column $j$. When working over some field $k$ we will define the residue $\operatorname{res}(b)$ associated to $b$ to be the image of $\operatorname{con}(b)$ under the standard map from $\mathbb{Z}$ to $k$.

For a partition $\lambda \vdash n$, a standard tableau of shape $\lambda$ is a numbering of the boxes of the Young diagram of $\lambda$ with the numbers $1,2, \ldots, n$ in such a way that the numbers are increasing along the rows and down the columns of $\lambda$. We denote the set of all standard tableaux of shape $\lambda$ by $\mathcal{T}_{\lambda}$.

Representations of the symmetric group. Let $k$ be a field of characteristic $p \geq 0$. Let $\mathrm{H}_{n}$ (or just $\mathrm{H}$ when this will not cause confusion) be the group algebra $k \mathfrak{S}_{n}$. There are several different standard (and cellular) bases for $\mathrm{H}_{n}$ available in the literature. Here we will use the Murphy basis introduced in [Mur95] and follow the exposition given in [Mat99, Chapter 3]. For each partition $\lambda \vdash n$ and each pair of standard tableaux $\left(T_{1}, T_{2}\right) \in \mathcal{T}_{\lambda} \times \mathcal{T}_{\lambda}$ Murphy defined an element $m_{T_{1}, T_{2}}^{\lambda} \in \mathrm{H}_{n}$. (In fact these are defined over $\mathbb{Z} \mathfrak{S}_{n}$.) These elements form a standard basis for $\mathrm{H}_{n}$. We will not need the explicit construction of the Murphy basis elements for this paper and so will only recall some of their properties. Following Section 3, for any $\lambda \vdash n$ we define

$$
\begin{gathered}
\mathrm{H}^{\unrhd \lambda}=\left\langle m_{T_{1}, T_{2}}^{\mu},\left(T_{1}, T_{2}\right) \in \mathcal{T}_{\mu} \times \mathcal{T}_{\mu}, \mu \vdash n \text { with } \mu \unrhd \lambda\right\rangle, \quad \text { and } \\
\mathrm{H}^{\triangleright \lambda}=\left\langle m_{T_{1}, T_{2}}^{\mu},\left(T_{1}, T_{2}\right) \in \mathcal{T}_{\mu} \times \mathcal{T}_{\mu}, \mu \vdash n \text { with } \mu \triangleright \lambda\right\rangle .
\end{gathered}
$$

Then we have

$$
\mathrm{H}^{\lambda}=\mathrm{H}^{\unrhd \lambda} / \mathrm{H}^{\triangleright \lambda} \cong S^{\lambda} \otimes\left(S^{\lambda}\right)^{\text {op }}
$$

as H-bimodules and for any fixed $T_{1} \in \mathcal{T}_{\lambda}$ the set $m_{T, T_{1}}^{\lambda}$ for all $T \in \mathcal{T}_{\lambda}$ form a basis for the module $S^{\lambda}$. In fact, the modules $S^{\lambda}$ for $\lambda \vdash n$ are the familiar dual Specht modules for $\mathrm{H}_{n}$.

There is a bilinear form defined on each dual Specht module $S^{\lambda}$ and if we consider the quotient of $S^{\lambda}$ by the radical of its bilinear form then we have that

$$
S^{\lambda} / \operatorname{rad} S^{\lambda} \neq 0 \text { if and only if } \lambda \text { is } p \text {-restricted }
$$

where $p \geq 0$ is the characteristic of the field $k$. Moreover the set of all $D^{\lambda}:=S^{\lambda} / \operatorname{rad} S^{\lambda}$ for $\lambda$ running over the set of $p$-restricted partitions of $n$ form a complete set of pairwise non-isomorphic simple $\mathrm{H}_{n}$-modules. 
The blocks of $\mathrm{H}_{\mathrm{n}}$ are described by Nakayama's conjecture (see [JK81, Theorem 6.1.21]) which states that two partitions of $n$ are in the same block if and only if they have the same $p$ core. In particular, if a partition $\lambda$ is itself a $p$-core then it is alone in its block and we have $S^{\lambda}=D^{\lambda}$.

Define $\iota: \mathrm{H}_{n} \rightarrow \mathrm{H}_{n}$ to be the anti-automorphism defined by $\iota(x)=x^{-1}$ for all $x \in \mathfrak{S}_{n}$. Define also $\alpha: \mathrm{H}_{n} \rightarrow \mathrm{H}_{n}$ to be the automorphism defined by $\alpha(x)=(-1)^{\ell(x)} x$ for all $x \in \mathfrak{S}_{n}$. Note that, when restricted to $\mathrm{H}_{n}$, the anti-automorphism $\phi$ given in Definition 2.3 can be factorised as $\phi=\alpha \iota$.

Using the anti-automorphism $\iota$, the linear dual $M^{*}$ of any left $\mathrm{H}_{n}$-module can be given the structure of left $\mathrm{H}_{n}$-module by setting $(x \gamma)(m)=\gamma(\iota(x) m)$ for all $\gamma \in M^{*}, m \in M$ and $x \in \mathrm{H}_{n}$. This duality fixes every simple module, so we have $\left(D^{\lambda}\right)^{*} \cong D^{\lambda}$ for any $p$-restricted partition $\lambda$ (see [Mat99, Exercise 2.7]).

We also have another functor on $\mathrm{H}_{n}$-mod given by tensoring with the 1-dimensional sign representation sgn of $\mathrm{H}_{n}$. It is well known that $S^{\lambda} \otimes \operatorname{sgn} \cong\left(S^{\lambda^{T}}\right)^{*}$ for any partition $\lambda \vdash n$ (see for example [Mat99, Exercise 3.14(iii)]). For each $p$-restricted partition $\lambda$, the module $D^{\lambda} \otimes \operatorname{sgn}$ is also simple and so it must be isomorphic to some $D^{\mu}$ for some $p$-restricted partition $\mu$. We call the partition $\mu$ the Mullineux conjugate of the partition $\lambda$, and denoted by $\mu=\lambda^{M}$. The map $-{ }^{M}$ gives an involution on the set of $p$-restricted partitions of $n$. Note that in the literature, the Mullineux map is often defined as an involution $m$ on the set of $p$-regular partitions. Our Mullineux conjugate $\lambda^{M}$ is related to the involution $m$ simply by $\lambda^{M}=\left(m\left(\lambda^{T}\right)\right)^{T}$. There are several combinatorial descriptions of this involution, the first one given by Mullineux in [Mul79], but we will not need any explicit description for this paper. We only note that if $S^{\lambda}=D^{\lambda}$ then we have $D^{\lambda} \otimes \operatorname{sgn}=S^{\lambda} \otimes \operatorname{sgn} \cong\left(S^{\lambda^{T}}\right)^{*} \cong D^{\lambda^{T}}$ and hence $\lambda^{M}=\lambda^{T}$ in this case. This happens for instance when $\lambda$ is a $p$-core.

We finish this section with a couple of properties of the Murphy basis which we will need later in the paper.

Proposition 4.1. The Murphy basis has the following properties.

(1) $\iota\left(m_{T_{1}, T_{2}}^{\lambda}\right)=m_{T_{2}, T_{1}}^{\lambda}$ for all $T_{1}, T_{2} \in \mathcal{T}_{\lambda}, \lambda \vdash n$.

(2) For each $\lambda \vdash n$ and $T_{1} \in \mathcal{T}_{\lambda}$, the set of all $\alpha\left(m_{T, T_{1}}^{\lambda}\right)$ with $T \in \mathcal{T}_{\lambda}$ spans an $H_{n}$-module isomorphic to $\left(S^{\lambda^{T}}\right)^{*}$.

Proof. For part (1), see [Mat99, Chapter 3, 3.20(1)]. For part (2), simply note that for any $w \in \mathfrak{S}_{n}$ we have

$$
\alpha\left(w m_{T, T_{1}}^{\lambda}\right)=\alpha(w) \alpha\left(m_{T, T_{1}}^{\lambda}\right)=(-1)^{\ell(w)} w \alpha\left(m_{T, T_{1}}^{\lambda}\right) .
$$

So we have

$$
w \alpha\left(m_{T, T_{1}}^{\lambda}\right)=(-1)^{\ell(w)} \alpha\left(w m_{T, T_{1}}^{\lambda}\right)
$$

Therefore we have

$$
\left\langle\alpha\left(m_{T, T_{1}}^{\lambda}\right) \mid T \in \mathcal{T}_{\lambda}\right\rangle \cong S^{\lambda} \otimes \operatorname{sgn} \cong\left(S^{\lambda^{T}}\right)^{*}
$$

Note that the set of all $\alpha\left(m_{T_{1}, T_{2}}^{\lambda}\right)$ for $\lambda \vdash n, T_{1}, T_{2} \in \mathcal{T}_{\lambda}$ form another standard basis for $\mathrm{H}_{n}$. 


\section{Standard BASis AND Standard modules for the Periplectic Brauer Algebra}

In this section we will continue to consider an arbitrary field $k$ of characteristic $p \geq 0$. Let $0 \leq t \leq n$ with $n-t \in 2 \mathbb{Z}$ and define $I(n, t)$ to be the set of $(n, t)$-Brauer diagrams with precisely $t$ non-crossing propagating lines. Following [GL96, Section 4], each $(n, n)$-Brauer diagram with $t$ propagating lines can be uniquely written as $S_{1} w S_{2}^{\text {op }}$ with $S_{1}, S_{2} \in I(n, t)$, and $w \in \mathfrak{S}_{t}$, where $S_{2}^{\text {op }}$ denotes the $(t, n)$-Brauer diagram obtained by flipping $S_{2}$ horizontally.

Theorem 5.1. Set

$$
\Lambda_{n}=\{\lambda \vdash t, 0 \leq t \leq n \text { with } n-t \in 2 \mathbb{Z}\}
$$

with partial order $\unlhd$. Define

$$
\mathcal{B}_{n}=\left\{C_{\left(S_{1}, T_{1}\right)\left(S_{2}, T_{2}\right)}^{\lambda}:=S_{1} m_{T_{1}, T_{2}}^{\lambda} S_{2}^{\mathrm{op}}\left|\lambda \in \Lambda_{n}, T_{1}, T_{2} \in \mathcal{T}_{\lambda}, S_{1}, S_{2} \in I(n, t), t=\right| \lambda \mid\right\} .
$$

Then $\left(A_{n}, \mathcal{B}_{n}\right)$ is a standardly based algebra.

Proof. The proof follows exactly the proof of (C1) and (C2) in [GL96, Theorem 4.10]. Note that, up to a sign, the multiplication of diagrams in $A_{n}$ is the same as in the Brauer algebra (with parameter 0) and the sign does not affect the arguments as we consider linear spans of diagrams.

Following Section 3 we have a filtration of the periplectic Brauer algebra $A_{n}$ with sections

$$
A_{n}^{\lambda} \cong W_{n}(\lambda) \otimes W_{n}^{\mathrm{op}}(\lambda)
$$

for each $\lambda \in \Lambda_{n}$. The left (respectively right) standard module $W_{n}(\lambda)$ (respectively $W_{n}^{\mathrm{op}}(\lambda)$ ) is spanned by the elements $C_{(S, T)\left(S_{1}, T_{1}\right)}^{\lambda}$ (respectively $\left.C_{\left(S_{1}, T_{1}\right)(S, T)}^{\lambda}\right)$ for all $S \in I(n, t), T \in \mathcal{T}_{\lambda}$ and some fixed $S_{1} \in I(n, t)$ and $T_{1} \in \mathcal{T}_{\lambda}$.

Note that if we used the basis $\alpha\left(m_{T_{1}, T_{2}}^{\lambda}\right)$ instead of $m_{T_{1}, T_{2}}^{\lambda}$ to construct the standard basis for $A_{n}$, we would get a different set of standard modules, which we denote by $\widetilde{W}_{n}(\lambda)$, spanned by $\widetilde{C}_{(T, S)\left(T_{1} S_{1}\right)}^{\lambda}=S \alpha\left(m_{T, T_{1}}^{\lambda}\right) S_{1}^{o p}$.

Remark 5.2. When $|\lambda|=n$ we see that $C_{(S, T)\left(S_{1}, T_{1}\right)}^{\lambda}=m_{T T_{1}}^{\lambda}$ as $I(n, n)$ only contains the identity element. So in this case we have $W_{n}(\lambda)=S^{\lambda}$ inflated to $A_{n}$ using the surjection $A_{n} \rightarrow \mathrm{H}_{n}$ which maps any diagram with at least one cup (or cap) to 0.

More generally, it is clear from the definition of $W_{n}(\lambda)$ given above that we can write it as

$$
W_{n}(\lambda)=V(n, t) \otimes_{\mathfrak{S}_{t}} S^{\lambda}
$$

where $V(n, t)$ is the span of all $(n, t)$-Brauer diagrams with exactly $t$ propagating lines and the action of $A_{n}$ on $V(n, t) \otimes_{\mathfrak{S}_{t}} S^{\lambda}$ is given as follows. Let $d$ be an $(n, n)$-Brauer diagram, $S$ be a $(n, t)$-Brauer diagram with $t$ propagating lines and $x \in S^{\lambda}$. Consider the multiplication $d S$ as defined in (1). If $d \star S$ has fewer than $t$ propagating lines then we set $d(S \otimes x)=0$. Otherwise we set $d(S \otimes x)=d S \otimes x$.

Similarly, using Proposition 4.1 (2), we have that

$$
\widetilde{W}_{n}(\lambda)=V(n, t) \otimes_{\mathfrak{S}_{t}}\left(S^{\lambda^{T}}\right)^{*} .
$$

In fact, we have that $V(n, t) \otimes_{\mathfrak{S}_{t}}-$ gives a functor from $\mathrm{H}_{t}$-mod to $A_{n}$-mod. It is easy to see that $V(n, t)$ is projective as a right $\mathfrak{S}_{t}$-module and so this functor is exact. Now as $\left(D^{\lambda}\right)^{*} \cong D^{\lambda}$ 
for all simple $\mathrm{H}_{t}$-modules we have that $\left(S^{\lambda^{T}}\right)^{*}$ and $S^{\lambda^{T}}$ have the same composition factors. The next proposition then follows immediately from the exactness of the functor $V(n, t) \otimes_{\mathfrak{S}_{t}}-$.

Proposition 5.3. For any $\lambda \in \Lambda_{n}$ the $A_{n}$-modules $\widetilde{W}_{n}(\lambda)$ and $W_{n}\left(\lambda^{T}\right)$ have the same composition factors.

Recall the definition of the anti-automorphism $\phi$ given in Definition 2.3.

Proposition 5.4. Let $\lambda \in \Lambda_{n}$. We have

$$
\phi\left(W_{n}^{\mathrm{op}}(\lambda)\right) \cong \widetilde{W}_{n}(\lambda) .
$$

Proof. We have that $\phi\left(W_{n}^{\mathrm{op}}(\lambda)\right)$ is spanned by the set of all $\phi\left(C_{\left(T_{1}, S_{1}\right)(T, S)}^{\lambda}\right)$ for $(T, S) \in \mathcal{T}_{\lambda} \times$ $I(n, t)$. Now we have

$$
\begin{aligned}
\phi\left(C_{\left(T_{1}, S_{1}\right),(T, S)}^{\lambda}\right) & =\phi\left(S_{1} m_{T_{1}, T}^{\lambda} S^{\mathrm{op}}\right) \\
& = \pm S \phi\left(m_{T_{1}, T}^{\lambda}\right)\left(S_{1}\right)^{\mathrm{op}} \\
& = \pm S \alpha\left(m_{T, T_{1}}^{\lambda}\right)\left(S_{1}\right)^{\mathrm{op}} \\
& \left.= \pm \widetilde{C}_{(T, S),\left(T_{1}, S_{1}\right)}^{\lambda}\right) .
\end{aligned}
$$

Here we used Proposition 4.1 (1) and the fact that, when restricted to the symmetric group, $\phi=\alpha \iota$. These elements span the left $A_{n}$-module $\widetilde{W}_{n}(\lambda)$ by definition.

Using the anti-automorphism $\phi$, we can define a contravariant exact functor

$$
\Upsilon: A_{n}-\bmod \rightarrow A_{n} \text {-mod; } \quad M \mapsto M^{*}=\operatorname{Hom}_{\mathbb{k}}(M, \mathbb{k}),
$$

where the $A_{n}$ action on $M^{*}$ is given by

$$
a \gamma(m):=\gamma(\phi(a) m), \text { for all } a \in A_{n}, \gamma \in M^{*}, m \in M .
$$

The functor $\Upsilon$ gives a contravariant equivalence of categories. This implies the following result.

Proposition 5.5. Let $\lambda \in \Lambda_{n}$. All composition factors of $\Upsilon\left(W_{n}(\lambda)\right)$ belong to the same block.

Proof. As $\Upsilon$ is a contravariant equivalence of categories, this follows directly from the fact that all composition factors of $W_{n}(\lambda)$ belong to the same block, as discussed at the end of Section 3 .

Now define

$$
\Lambda_{n}^{\prime}=\left\{\lambda \in \Lambda_{n}: \lambda \text { is } p \text {-restricted and } \lambda \neq \emptyset \text { when } n \text { is even }\right\} .
$$

It was shown in [Cou18] that the set of $L_{n}(\lambda):=W_{n}(\lambda) / \operatorname{rad} W_{n}(\lambda)$ for all $\lambda \in \Lambda_{n}^{\prime}$ form a complete set of pairwise non-isomorphic simple $A_{n}$-modules. For each $\lambda \in \Lambda_{n}^{\prime}$ we denote by $P_{n}(\lambda)$ the projective cover of $L_{n}(\lambda)$.

We will later wish to consider reduction $\bmod p$ from characteristic 0 to characteristic $p$. Notice that the basis in Theorem 5.1 is defined over $R$, and we will later use this to relate the standard modules over $\mathbb{K}$ to those over $\mathbb{k}$. When we wish to emphasise the choice of field we will use a superscript; for example $W_{n}^{\mathbb{K}}(\lambda)$ will denote the standard module defined over $\mathbb{K}$. 


\section{LOCALISATION AND GLOBALISATION FUNCTORS}

In this section we work over an arbitrary field $k$. Recall that the symmetric group algebra $\mathrm{H}_{n}$ is the quotient of $A_{n}$ with basis consisting of Brauer diagrams without cups or caps. We can extend every $\mathrm{H}_{n}$-module $M$ to an $A_{n}$-module by letting the Brauer diagrams which contains cups and caps act trivially. This gives an embedding of the category $\mathrm{H}_{n}$-mod into the category $A_{n}$-mod.

We can also embed $A_{n}$-mod in $A_{n+2}$-mod for $n \geq 1$. For this we need the idempotent $\epsilon_{n+2}$ which has $n-1$ non-crossing propagating lines joining the left-most nodes, a propagating line joining the third northern node from the right with the right-most southern node, one cup joining the two right-most northern nodes and one cap joining the penultimate southern node with its left neighbour, as illustrated in Figure 4.

$$
\epsilon_{n+2}=\quad|\cdots|
$$

Figure 4. An idempotent in $A_{n+2}$

We also introduce the $(n+2, n)$-Brauer diagram $g_{n+2, n}$ which has $n$ non-crossing propagating lines connecting the left-most nodes and one cup connecting the remaining northern nodes and the $(n, n+2)$-Brauer diagram $f_{n, n+2}$ which has $n-1$ non-crossing propagating lines connecting the left-most nodes, one propagating line connecting the right-most nodes and one cap connecting the remaining southern nodes. We illustrate these two diagrams in Figures 5 and 6 . It is clear that $f_{n, n+2} g_{n+2, n}=\mathrm{id}_{n}$ and $g_{n+2, n} f_{n, n+2}=\epsilon_{n+2}$.

$$
g_{n+2, n}=|\cdots|+\mid
$$

FiguRE 5. The $(n+2, n)$-Brauer diagram $g_{n+2, n}$

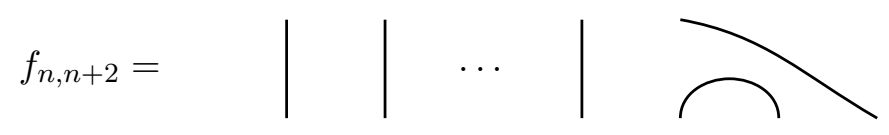

FiguRE 6 . The $(n, n+2)$-Brauer diagram $f_{n, n+2}$

One can then easily verify the following lemma.

Lemma 6.1. Let $n \geq 1$. There is an algebra isomorphism

$$
A_{n} \rightarrow \epsilon_{n+2} A_{n+2} \epsilon_{n+2},
$$

which maps $a \in A_{n}$ to $g_{n+2, n} a f_{n, n+2}$. The inverse maps $b \in \epsilon_{n+2} A_{n+2} \epsilon_{n+2}$ to $f_{n, n+2} b g_{n+2, n}$.

We define a functor $F_{n+2}: A_{n+2}$-mod $\rightarrow A_{n}$-mod by mapping $M$ to $\epsilon_{n+2} M$ and using the isomorphism of Lemma 6.1. We also define

$$
\begin{aligned}
G_{n}: A_{n}-\bmod & \rightarrow A_{n+2}-\bmod \\
M & \mapsto A_{n+2} \epsilon_{n+2} \otimes_{A_{n}} M .
\end{aligned}
$$


It is clear that $F_{n+2} G_{n}(M) \cong M$ for all $A_{n}$-modules $M$. Hence $G_{n}$ gives an embedding of $A_{n}$-mod in $A_{n+2}$-mod.

The functors $F_{n}$ and $G_{n}$ as defined above are analogues of corresponding functors for the ordinary Brauer algebra considered in [CDM09a] and [DWH99]. As vector spaces, the standard modules $W(\lambda)$ for the periplectic Brauer algebra are isomorphic to the corresponding standard modules $\Delta(\lambda)$ for the Brauer algebra, and so it is easy to see that the arguments in [CDM09a] and [DWH99] generalise to the periplectic case to give the following result.

Lemma 6.2. For $n \geq 1$ and $\lambda \in \Lambda_{n}$ we have

$$
G_{n}\left(W_{n}(\lambda)\right)=W_{n+2}(\lambda) .
$$

It follows immediately that for $n \geq 3$ and $\lambda \in \Lambda_{n}$ we have

$$
F_{n}\left(W_{n}(\lambda)\right) \cong \begin{cases}W_{n-2}(\lambda) & \text { if } \lambda \in \Lambda_{n-2} \\ 0 & \text { otherwise }\end{cases}
$$

and from the exactness of $F_{n}$ that for $\lambda \in \Lambda_{n}^{\prime}$ we have

$$
F_{n}\left(L_{n}(\lambda)\right) \cong \begin{cases}L_{n-2}(\lambda) & \text { if } \lambda \in \Lambda_{n-2}^{\prime} \\ 0 & \text { otherwise. }\end{cases}
$$

By induction and exactness of $F_{n}$ we obtain the following result.

Lemma 6.3. Let $\lambda \in \Lambda_{n}$ and $\mu \in \Lambda_{n}^{\prime}$. Then

$$
\left[W_{n}(\lambda): L_{n}(\mu)\right]= \begin{cases}{\left[W_{|\mu|}(\lambda): L_{|\mu|}(\mu)\right]} & \text { if }|\lambda| \leq|\mu|, \\ 0 & \text { if }|\lambda|>|\mu| .\end{cases}
$$

\section{BGG RECIPROCITY IN ARBITRARY CHARACTERISTIC}

We continue to work over an arbitrary field $k$ of characteristic $p \geq 0$. In the last Section we saw that certain aspects of the representation theories of the periplectic and Brauer algebras are very similar. In contrast, the results in this Section begin to illustrate their striking differences.

Proposition 7.1. For any $\lambda \in \Lambda_{n}^{\prime}$ we have

$$
\Upsilon\left(L_{n}(\lambda)\right) \cong L_{n}\left(\lambda^{M}\right)
$$

where $\lambda^{M}$ denotes the Mullineux conjugate of the partition $\lambda$.

Proof. We prove this by induction on $n$. If $n=0$ or 1 there is nothing to prove as $A_{0}=A_{1}=\mathbb{k}$ and there is only one simple module. Let $n \geq 2$. If $|\lambda|=n$ then $L_{n}(\lambda)=D^{\lambda}$ (lifted to $A_{n}$ ). In this case we have $\Upsilon\left(D^{\lambda}\right)=\left(D^{\lambda} \otimes \operatorname{sgn}\right)^{*}=\left(D^{\lambda^{M}}\right)^{*} \cong D^{\lambda^{M}}$ so we are done.

If $|\lambda| \leq n-2$ then $\Upsilon\left(L_{n}(\lambda)\right)$ is certainly a simple $A_{n}$-module. Applying the localisation functor, we get the $A_{n-2}$-module $F_{n}\left(\Upsilon\left(L_{n}(\lambda)\right)=\epsilon_{n} \Upsilon\left(L_{n}(\lambda)\right)\right.$, where we use the isomorphism $A_{n-2} \cong$ $\epsilon_{n} A_{n} \epsilon_{n}$ from Lemma 6.1. We have

$$
\epsilon_{n} \Upsilon\left(L_{n}(\lambda)\right)=\Upsilon\left(\phi\left(\epsilon_{n}\right) L_{n}(\lambda)\right) .
$$

Now $\phi\left(\epsilon_{n}\right) L_{n}(\lambda)$ is a simple $\phi\left(\epsilon_{n}\right) A_{n} \phi\left(\epsilon_{n}\right)$-module and $\phi\left(\epsilon_{n}\right) A_{n} \phi\left(\epsilon_{n}\right) \cong A_{n-2}$ (this can be seem by swapping the roles of $f$ and $g$ in Lemma 6.1). Using the corresponding localisation functor we get $\phi\left(\epsilon_{n}\right) L_{n}(\lambda) \cong L_{n-2}(\lambda)$. Finally, we obtain

$$
F_{n}\left(\Upsilon\left(L_{n}(\lambda)\right) \cong \Upsilon\left(L_{n-2}(\lambda)\right) \cong L_{n-2}\left(\lambda^{M}\right)\right.
$$


by induction. Thus we must have $\Upsilon\left(L_{n}(\lambda)\right) \cong L_{n}\left(\lambda^{M}\right)$ as required.

Lemma 7.2. Let $\lambda \in \Lambda_{n}^{\prime}$ and $e_{\lambda}$ be a primitive idempotent satisfying $P_{n}(\lambda)=A_{n} e_{\lambda}$. Then we have

$$
A_{n} \phi\left(e_{\lambda}\right)=P_{n}\left(\lambda^{M}\right)
$$

Proof. Clearly $\phi\left(e_{\lambda}\right)$ is a primitive idempotent. So $A_{n} \phi\left(e_{\lambda}\right)=P_{n}(\mu)$ for some $\mu \in \Lambda_{n}^{\prime}$. Now we have

$$
\begin{aligned}
\operatorname{Hom}_{A_{n}}\left(P_{n}(\mu), L_{n}\left(\lambda^{M}\right)\right) & \cong \operatorname{Hom}_{A_{n}}\left(A_{n} \phi\left(e_{\lambda}\right), \Upsilon\left(L_{n}(\lambda)\right)\right) \\
& \cong \phi\left(e_{\lambda}\right) \Upsilon\left(L_{n}(\lambda)\right) \\
& =\Upsilon\left(e_{\lambda} L_{n}(\lambda)\right) \\
& \cong \Upsilon\left(\operatorname{Hom}_{A_{n}}\left(A_{n} e_{\lambda}, L_{n}(\lambda)\right)\right. \\
& =\Upsilon\left(\operatorname{Hom}_{A_{n}}\left(P_{n}(\lambda), L_{n}(\lambda)\right)\right. \\
& =\Upsilon(\mathbb{k}) \cong \mathbb{k} .
\end{aligned}
$$

This shows that we must have $\mu=\lambda^{M}$ as required.

The following theorem generalises the BGG-reciprocity given in [Cou18, Theorem 3] to field of arbitrary characteristics.

Theorem 7.3. Let $\lambda \in \Lambda_{n}^{\prime}$. There is a filtration of the projective indecomposable module $P_{n}(\lambda)$ by standard modules and if we denote by $\left(P_{n}(\lambda): W_{n}(\mu)\right)$ the number of subquotients in this filtration which are isomorphic to $W_{n}(\mu)$ for $\mu \in \Lambda_{n}$ then we have

$$
\left(P_{n}(\lambda): W_{n}(\mu)\right)=\left[W_{n}\left(\mu^{T}\right): L_{n}\left(\lambda^{M}\right)\right]
$$

where $\mu^{T}$ denotes the transpose of the partition $\mu$ and $\lambda^{M}$ denotes the Mullineux conjugate of the partition $\lambda$.

(Note that when $p>n$ or $p=0$ we have that $\Lambda_{n}^{\prime}=\Lambda_{n} \backslash\{\varnothing\}$ and $\lambda^{M}=\lambda^{T}$ for all $\lambda \in \Lambda_{n}$.)

Proof. Using Proposition 3.1 (2) we have that

$$
\left(P_{n}(\lambda): W_{n}(\mu)\right)=\operatorname{dim}\left(W_{n}^{\mathrm{op}}(\mu) \otimes_{A_{n}} P_{n}(\lambda)\right) .
$$

Now we have

$$
\begin{aligned}
\operatorname{dim}\left(W_{n}^{\mathrm{op}}(\mu) \otimes_{A_{n}} P_{n}(\lambda)\right) & =\operatorname{dim}\left(W_{n}^{\mathrm{op}}(\mu) \otimes_{A_{n}} A_{n} e_{\lambda}\right) \\
& =\operatorname{dim} W_{n}^{\mathrm{op}}(\mu) e_{\lambda} \\
& =\operatorname{dim} \phi\left(W_{n}^{\mathrm{op}}(\mu) e_{\lambda}\right) \\
& =\operatorname{dim} \phi\left(e_{\lambda}\right) \phi\left(W_{n}^{\mathrm{op}}(\mu)\right) \\
& =\operatorname{dim} \phi\left(e_{\lambda}\right) \widetilde{W}_{n}(\mu)
\end{aligned}
$$

using Proposition 5.4. Then we get

$$
\begin{aligned}
\operatorname{dim} \phi\left(e_{\lambda}\right) \widetilde{W}_{n}(\mu) & =\operatorname{dim} \operatorname{Hom}_{A_{n}}\left(A_{n} \phi\left(e_{\lambda}\right), \widetilde{W}_{n}(\mu)\right. \\
& =\operatorname{dim} \operatorname{Hom}_{A_{n}}\left(P\left(\lambda^{M}\right), \widetilde{W}_{n}(\mu)\right) \\
& =\left[\widetilde{W}_{n}(\mu): L_{n}\left(\lambda^{M}\right)\right]
\end{aligned}
$$

using Lemma 7.2. Finally, using Proposition 5.3, we get that

$$
\left[\widetilde{W}_{n}(\mu): L_{n}\left(\lambda^{M}\right)\right]=\left[W_{n}\left(\mu^{T}\right): L_{n}\left(\lambda^{M}\right)\right]
$$


as required.

As a consequence of this BGG-reciprocity, we obtain the following factorisation of the Cartan matrix of the periplectic Brauer algebra $A_{n}$ in arbitrary characteristic.

Corollary 7.4. For $\lambda, \nu \in \Lambda_{n}^{\prime}$ and $\mu \in \Lambda_{n}$ define the composition multiplicities

$$
C_{\lambda \nu}=\left[P_{n}(\lambda): L_{n}(\nu)\right] \quad \text { and } \quad D_{\mu \nu}=\left[W_{n}(\mu): L_{n}(\nu)\right] .
$$

Then we have

$$
C_{\lambda \nu}=\sum_{\mu \in \Lambda_{n}} D_{\mu^{T} \lambda^{M}} D_{\mu \nu}
$$

\section{Blocks IN CHARACTERISTIC $p>2$}

Recall from Section 3 that we can define the blocks of $A_{n}$ as equivalence classes on $\Lambda_{n}$. In [Cou18], Coulembier described the blocks of the periplectic Brauer algebra $A_{n}$ when the characteristic $p$ of the field satisfies $p \notin[2, n]$. We recall his result below.

Theorem 8.1. [Cou18, Theorem 1] Let $A_{n}$ be the periplectic Brauer algebra over a field of characteristic $p \notin[2, n]$. Let $\lambda, \mu \in \Lambda_{n}$. Then $\lambda$ and $\mu$ are in the same $A_{n}$-block if and only if they have the same 2-core.

The aim of this section is to generalise this result to fields of characteristic $p>2$. As described in the introduction we will fix a $p$-modular system $(\mathbb{K}, R, \mathbb{k})$ with char $\mathbb{k}=p>2$. Throughout this section we consider the periplectic Brauer algebra $A_{n}$ over the field $\mathbb{k}$. Unless otherwise indicated by a superscript, all the $A_{n^{-}}$modules considered are therefore over the field $\mathbb{k}$.

Proposition 8.2. Let $\lambda, \mu \in \Lambda_{n}$. If $|\lambda|=|\mu|$ and $\lambda$ and $\mu$ have the same $p$-core then $\lambda$ and $\mu$ are in the same $A_{n}$-block.

Proof. We know that if $\lambda$ and $\mu$ have the same $p$-core, then $S^{\lambda}$ and $S^{\mu}$ belong to the same $\mathbb{k} \mathfrak{S}_{|\lambda|}$-block by Nakayama's conjecture [JK81, Theorem 6.1.21]. Using Remark 5.2 we deduce that $W_{|\lambda|}(\lambda)$ and $W_{|\lambda|}(\mu)$ belong to the same $A_{n}$-block. Now, by repeated application of the globalisation functors $G_{n-2} \ldots G_{|\lambda|+2} G_{|\lambda|}$ and Lemma 6.2 we deduce that $W_{n}(\lambda)$ and $W_{n}(\mu)$ belong to the same $A_{n}$-blocks.

Proposition 8.3. Let $\lambda$ and $\mu$ be in $\Lambda_{n}$. Then we have that $\lambda$ and $\mu$ are in the same $A_{n}$-block if and only if $\lambda^{T}$ and $\mu^{T}$ are in the same $A_{n}$-block.

Proof. First suppose that $\lambda$ and $\mu$ are in $\Lambda_{n}^{\prime}$. The simple modules $L_{n}(\lambda)$ and $L_{n}(\mu)$ are in the same block if and only if $\Upsilon\left(L_{n}(\lambda)\right)$ and $\Upsilon\left(L_{n}(\mu)\right)$ are in the same block. But we have $\Upsilon\left(L_{n}(\lambda)\right) \cong L_{n}\left(\lambda^{M}\right)$ and $\Upsilon\left(L_{n}(\mu)\right) \cong L_{n}\left(\mu^{M}\right)$ by Proposition 7.1. Now for any $p$-restricted partition $\nu$ we have that $\nu^{T}$ and $\nu^{M}$ belong to the same block. This follows from the fact that $S^{\nu} \otimes \operatorname{sgn} \cong\left(S^{\nu^{T}}\right)^{*}$ and $D^{\nu} \otimes \operatorname{sgn} \cong D^{\nu^{M}}$, together with Corollary 3.2 and the fact that duality fixes simple modules for the symmetric group. Thus we can conclude that $\lambda$ and $\mu$ belong to the same block if and only if $\lambda^{T}$ and $\mu^{T}$ belong to the same block.

Now suppose that $\lambda$ and $\mu$ are general. Choose $\lambda^{\prime}$ and $\mu^{\prime}$ in $\Lambda_{n}^{\prime}$ such that $\lambda^{\prime}$ has the same $p$-core as $\lambda$ (respectively $\mu^{\prime}$ has the same $p$-core as $\mu$ ), and $\left|\lambda^{\prime}\right|=|\lambda|$ (respectively $\left.\left|\mu^{\prime}\right|=|\mu|\right)$ ). These must exist by considering composition factors of the associated Specht modules and using Proposition 8.2. Further we have that $\left(\lambda^{\prime}\right)^{T}$ has the same $p$-core as $\lambda^{T}$ and $\left(\mu^{\prime}\right)^{T}$ has the same 
$p$-core as $\mu^{T}$. Therefore $\lambda$ is in the same block as $\lambda^{\prime}$ and $\lambda^{T}$ is in the same block as $\left(\lambda^{\prime}\right)^{T}$ (and similarly for $\mu$ ) by Proposition 8.2. The result now follows from the first case considered above.

We will need the following well-known lemma.

Lemma 8.4. [Kin14, Lemma 4.1] Let $A$ be an R-algebra which is free of finite rank as an $R$-module. Suppose that $X$ and $Y$ are $R$-free $A$-modules of finite rank and let $M \subseteq \mathbb{K} Y$. If $\operatorname{Hom}_{\mathbb{K} A}(\mathbb{K} X, \mathbb{K} Y / M) \neq 0$ then there is a submodule $N \subseteq \mathbb{k} Y$ such that $\operatorname{Hom}_{\mathbb{k} A}(\mathbb{k} X, \mathbb{k} Y / N) \neq 0$. Moreover, $N$ can be taken to be the p-modular reduction of an $R$-lattice in $M$.

Proposition 8.5. If $\lambda$ can be obtained from $\mu \in \Lambda_{n}$ by removing two boxes in the same row (respectively column), then $\lambda$ and $\mu$ belong to the same $A_{n}$-block.

Proof. By Proposition 8.3 it is enough to consider the case when $\lambda$ is obtained from $\mu$ by removing two boxes in the same row. For a field $\mathbb{K}$ of characteristic zero, we have [Cou18, Proposition $7.2 .6]$

$$
\left[W_{n}^{\mathbb{K}}(\lambda): L_{n}^{\mathbb{K}}(\mu)\right]=1 .
$$

This implies that there exists a submodule $M$ of $W_{n}^{\mathbb{K}}(\lambda)$ such that

$$
\operatorname{Hom}_{A_{n}^{\mathbb{K}}}\left(W_{n}^{\mathbb{K}}(\mu), W_{n}^{\mathbb{K}}(\lambda) / M\right) \neq 0 .
$$

By Lemma 8.4, we can reduce this modulo $p$ to obtain

$$
\operatorname{Hom}_{A_{n}^{\mathrm{k}}}\left(W_{n}^{\mathbb{k}}(\mu), W_{n}^{\mathbb{k}}(\lambda) / \bar{M}\right) \neq 0,
$$

where $\bar{M}$ is a submodule of $W_{n}^{\mathbb{k}}(\lambda)$. In particular $\mu$ and $\lambda$ belong to the same block.

We say that $\mathfrak{t}=\left(\mathfrak{t}^{(1)}, \mathfrak{t}^{(2)}, \ldots, \mathfrak{t}^{(n)}\right)$ is a path of partitions if each $\mathfrak{t}^{(i)}$ is a partition such that $\mathfrak{t}^{(i+1)}$ is obtained from $\mathfrak{t}^{(i)}$ by adding or removing one box in the Young diagram and $\mathfrak{t}^{(1)}=(\square)$. We denote the set of all paths of length $n$ with $\mathfrak{t}^{(n)}=\lambda$ by $S t_{n}(\lambda)$. We define the vector $c_{\mathfrak{t}}=\left(c_{\mathfrak{t}}(2), c_{\mathfrak{t}}(3), \ldots, c_{\mathfrak{t}}(n)\right) \in \mathbb{k}^{n-1}$ for $\mathfrak{t} \in S t_{n}(\lambda)$ as follows

$$
c_{\mathfrak{t}}(i)= \begin{cases}\operatorname{res}(b) & \text { if } \mathfrak{t}^{(i)}=\mathfrak{t}^{(i-1)} \cup b \\ \operatorname{res}(b)+1 & \text { if } \mathfrak{t}^{(i)} \cup b=\mathfrak{t}^{(i-1)},\end{cases}
$$

where $b$ is the box added to or removed from $\mathfrak{t}^{(i-1)}$ to obtain $\mathfrak{t}^{(i)}$.

We will need the following pair of lemmas.

Lemma 8.6 ([Cou18, Proposition 6.2.6]). For $\lambda \in \Lambda_{n}$ and $\mu \in \Lambda_{n}^{\prime}$, if $\left[W_{n}(\lambda): L_{n}(\mu)\right] \neq 0$ then there exist $\mathfrak{t} \in S t_{n}(\lambda)$ and $\mathfrak{s} \in S t_{n}(\mu)$ such that $c_{\mathfrak{t}}=c_{\mathfrak{s}}$.

Lemma 8.7. Assume $\lambda \in \Lambda_{n}$ has as 2 -core an $r$-staircase with $2 r-1<p$, and that

$$
\frac{r(r+1)}{2}+p-2 r>n
$$

Then $\lambda$ is a p-core and every partition $\mu$ obtained by adding a box to $\lambda$ is still a p-core. Further, two boxes in $\mu$ (or in $\lambda$ ) have the same residue if and only if they have the same content.

Proof. The degree of an $r$-staircase is $\frac{r(r+1)}{2}$ and the length of its rim is $2 r-1$. The partition $\lambda$ is obtained by adding at most $p-2 r-1$ boxes to the $r$-staircase since $\frac{r(r+1)}{2}+p-2 r>n$. Therefore the length of the rim of $\lambda$ is at most $2 r-1+p-2 r-1=p-2$. In particular $\lambda$ is a $p$-core and this is still true for the partition $\mu$ since the length of the rim will still be smaller 
than $p$. As the possible contents occuring in $\mu$ all occur in the rim, and these boxes all have different residues, the residues in $\mu$ for boxes with differing contents must be distinct.

Proposition 8.8. Consider $\mu \in \Lambda_{n}^{\prime}$ such that the 2-core of $\mu$ is given by an r-staircase with $2 r-1<p$ and $\frac{r(r+1)}{2}+p-2 r>n$. Then for all $\lambda \in \Lambda_{n}$ we have that

$$
\left[W_{n}(\lambda): L_{n}(\mu)\right] \neq 0
$$

implies $\lambda \subseteq \mu$ and $\lambda$ and $\mu$ have the same 2-core, and for all $\nu \in \Lambda_{n}^{\prime}$,

$$
\left[W_{n}(\mu): L_{n}(\nu)\right] \neq 0
$$

implies $\mu \subseteq \nu$ and $\mu$ and $\nu$ have the same 2-core.

Proof. Assume $\left[W_{n}(\lambda): L_{n}(\mu)\right] \neq 0$ for $\lambda \in \Lambda_{n}$. We may further assume $n=|\mu|$ by Lemma 6.3. From Lemma 8.6, it then follows that there exists $\mathfrak{t} \in S t_{n}(\lambda)$ and $\mathfrak{s} \in S t_{n}(\mu)$ such that $c_{\mathfrak{t}}=c_{\mathfrak{s}}$. Because $\mu \vdash n$, we only add boxes in $\mathfrak{s}$ and $c_{\mathfrak{s}}(i)$ is equal to the residue of the added box.

If $\lambda \nsubseteq \mu$, then there exists a box $b$ in $\lambda$ which is not contained in $\mu$ but such that $\mu \cup b$ is a partition. The residue of $b$ appears in $c_{\mathfrak{t}}$ and thus also in $c_{\mathfrak{s}}$. Therefore $\mu$ should also contain a box $b^{\prime}$ not in $\lambda$ with the same residue. But from Lemma 8.7 it follows that $b$ and $b^{\prime}$ must have the same content, which is clearly impossible as they would have to lie on the same diagonal and $b^{\prime}$ would then belong to $\lambda$ (since $\lambda$ is a partition). So we conclude that $\lambda \subseteq \mu$. Moreover, since the residues determines the contents for $\lambda$ and $\mu$ (by Lemma 8.7) it follows in the same way as in the characteristic zero situation that $\mu$ and $\lambda$ have the same 2-core, see [Cou18, Corollary 6.2.7 and Lemma 7.3.3].

Now assume $\left[W_{n}(\mu): L_{n}(\nu)\right] \neq 0$ for $\nu \in \Lambda_{n}^{\prime}$. By Lemma 6.3 , it again suffices to consider the case $n=|\nu|$. If $|\mu|=|\nu|$ it follows as in the previous case that the existence of a box in $\nu$ not contained in $\mu$ is impossible. So then $\mu=\nu$.

Consider $|\mu|<|\nu|$. We have $\mathfrak{t} \in S t_{n}(\nu)$ and $\mathfrak{s} \in S t_{n}(\mu)$ such that $c_{\mathfrak{t}}=c_{\mathfrak{s}}$ as follows from Lemma 8.6. Let $\kappa$ be the partition containing all the boxes which are added in $\mathfrak{s}$. In particular $\kappa$ contains $\mu$ and the 2 -core $(r, r-1, \ldots, 2,1)$ of $\mu$. If we would need to add more than $p-2 r-2$ boxes to obtain $\kappa$ from this 2-core, then $|\nu| \geq|\kappa|+1>p-2 r-2+\frac{r(r+1)}{2}+1 \geq n$. This is impossible since $\nu \vdash n$. Thus we add at most $p-2 r-2$ boxes to the $r$-staircase to obtain $\kappa$ and the length of the rim of $\kappa$ is smaller than or equal to $2 r-1+p-2 r-2=p-3$.

We claim that $\nu_{1} \leq \kappa_{1}+1$ and that $\kappa_{k}=0$ implies $\nu_{k+1}=0$. This can be seen as follows. If $\nu_{1}>\kappa_{1}+1$, then $\nu$ would contain a box in the first row which is two places to the right of the rightmost box of $\kappa$. The residue of this box is not equal to $\operatorname{res}(b)$ or $\operatorname{res}(b)+1$ for any box $b$ in $\kappa$ because the length of the rim of $\kappa$ is smaller than $p-2$. This is in contradiction with $c_{\mathfrak{t}}=c_{\mathfrak{s}}$. Similarly there can be no box of $\nu$ two rows under the last non-zero row of $\kappa$. These conditions on $\nu$ mean that the length of the rim of $\nu$ is smaller than $p$. Hence the contents of $\nu$ are determined by the residues. Then $\mu \subseteq \nu$ again implies that they have the same 2-core and $\mu \nsubseteq \nu$ is impossible because it would mean that $\mu$ contains a box with residue not occurring in $\nu$.

For each $\lambda \in \Lambda_{n}$, denote by $\Lambda_{n}(\lambda)$ the subset of partitions of $\Lambda_{n}$ which are in the same block as $\lambda$.

Proposition 8.9. Consider the r-staircase partition $\rho_{r}=(r, r-1, r-2, \ldots, 2,1)$ in $\Lambda_{n}$ where $r$ is such that $2 r-1<p$ and $\frac{r(r+1)}{2}+p-2 r>n$. Then $\lambda \in \Lambda_{n}\left(\rho_{r}\right)$ if and only if the 2-core of $\lambda$ is $\rho_{r}$. 
Proof. Assume that the 2-core of $\lambda \in \Lambda_{n}$ is $\rho_{r}$. Then we can find a chain of partitions

$$
\rho_{r}=\lambda^{(0)} \subset \lambda^{(1)} \subset \cdots \subset \lambda^{(l)}=\lambda,
$$

such that each $\lambda^{(i)}$ is obtained from $\lambda^{(i-1)}$ by adding a vertical or horizontal 2-hook. Then it follows from Proposition 8.5 that $\lambda$ and $\rho_{r}$ belong to the same block.

Now consider an arbitrary $\mu$ in $\Lambda_{n}^{\prime}$ with the 2-core of $\mu$ not equal to $\rho_{r}$. We will show that

$$
\left[P_{n}(\lambda): L_{n}(\mu)\right]=0 \text { and }\left[P_{n}(\mu): L_{n}(\lambda)\right]=0,
$$

for all $\lambda \in \Lambda_{n}^{\prime}$ which have as 2-core $\rho_{r}$. This implies that

$$
\Lambda_{n}\left(\rho_{r}\right)=\left\{\lambda \in \Lambda_{n} \mid \text { the } 2 \text {-core of } \lambda \text { is } \rho_{r}\right\} .
$$

Using Corollary 7.4, we find

$$
\left[P_{n}(\lambda): L_{n}(\mu)\right]=\sum_{\gamma \in \Lambda_{n}}\left[W_{n}\left(\gamma^{T}\right): L_{n}\left(\lambda^{M}\right)\right]\left[W_{n}(\gamma): L_{n}(\mu)\right] .
$$

Observe first that $\lambda^{M}=\lambda^{T}$, since $\lambda$ is a $p$-core by Lemma 8.7. Therefore $\lambda^{M}$ also has $\rho_{r}$ as 2-core. We know that $\left[W_{n}\left(\gamma^{T}\right): L_{n}\left(\lambda^{M}\right)\right]$ is non-zero only if $\gamma^{T}$ has the same 2-core as $\lambda^{M}$ from Proposition 8.8. This implies that $\gamma$ has $\rho_{r}$ as 2-core. But then $\left[W_{n}(\gamma): L_{n}(\mu)\right]$ is non-zero only if $\gamma$ and $\mu$ have the same 2-core, which is impossible since the 2-core of $\mu$ is not equal to $\rho_{r}$. So we conclude $\left[P_{n}(\lambda): L_{n}(\mu)\right]=0$. In a similar way we find $\left[P_{n}(\mu): L_{n}(\lambda)\right]=0$. This shows that $\mu$ is not in the same block as $\lambda$. Note that we also have that any $\mu \in \Lambda_{n}$ (not necessarily in $\Lambda_{n}^{\prime}$ ) with 2-core different from $\rho_{r}$ would also be in a different block from $\lambda$ using Proposition 8.8.

Proposition 8.10. Consider $\lambda \in \Lambda_{n}$ which has as 2-core an $r$-staircase with $2 r-1 \geq p$ or $\frac{r(r+1)}{2}+p-2 r \leq n$. Then $\lambda$ belongs to the same block as the empty partition $\varnothing$ if $n$ is even or to the same block as the partition (1) if $n$ is odd.

Proof. We will prove the proposition using induction on the number of boxes in the Young diagram corresponding to the partition. The induction base is trivially satisfied. We will now show that $\lambda$ is contained in the same block as a partition with contains two boxes fewer than $\lambda$ and which has as 2-core an $r^{\prime}$-staircase which also satisfies $2 r^{\prime}-1 \geq p$ or $\frac{r^{\prime}\left(r^{\prime}+1\right)}{2}+p-2 r^{\prime} \leq n$. By the induction hypothesis, it then follows that $\lambda$ is contained in the same block as $\varnothing$ if $n$ is even or as (1) if $n$ is odd.

First suppose that $\lambda$ is not a 2-core. Then there is a horizonal or vertical 2-hook which can be removed to leave a new partition $\mu$ with the same 2-core. By Proposition $8.5 \lambda$ and $\mu$ lie in the same block and $\mu$ has the same 2-core as $\lambda$.

Next suppose that $\lambda$ is a 2-core, but is not a $p$-core. Then $\lambda$ is an $r$-staircase and hence any removable rim $p$-hook cannot lie entirely in the first row. As a removable rim $p$-hook must exist, we can construct a new partition $\mu$ by removing from $\lambda$ this rim $p$-hook and adding $p$ boxes to the first row. Then $\lambda$ and $\mu$ belong to the same block by Proposition 8.2 since $\lambda$ and $\mu$ have the same $p$-core and $|\lambda|=|\mu|$. It is also clear that we can remove a horizontal 2-hook from the first row of $\mu$ to obtain a new partition $\nu$. Then Proposition 8.5 implies that $\lambda$ and $\nu$ are in the same block. Moreover, since $\mu$ has a removable rim $p$-hook, $\mu$, and thus also $\nu$, can not have a 2-core with $2 r-1<p$ and $\frac{r(r+1)}{2}+p-2 r>n$ because these conditions would imply that $\mu$ is a $p$-core by Lemma 8.7.

The only case we did not cover yet is when $\lambda$ is a $p$-core and a 2 -core at the same time. Since $\lambda$ is a 2 -core it is an $r$-staircase, i.e. $\lambda=(r, r-1, r-2, \ldots, 2,1)$. Because $\lambda$ is also a $p$-core it follows that $2 r-1<p$ and thus $2 r \leq p-1$ since $p$ is odd. Our condition on $\lambda$ then implies that 
$\frac{r(r+1)}{2}+p-2 r \leq n$. Note that this inequality is actually strict, since the parity of the left hand side is different from the parity of the right hand side. This follows because $\rho_{r}$ is contained in $\Lambda_{n}$, and therefore $\frac{r(r+1)}{2}$ and $n$ have the same parity. We can thus add $p-2 r+1$ boxes to the first row of $\lambda$ to obtain a new partition $\mu \in \Lambda_{n}$.

Now $\lambda$ and $\mu$ are in the same block by Proposition 8.5. Furthermore $\mu$ has a removable rim $p$-hook (consisting of all boxes on the rim of $\mu$ ) and the same $p$-core as the partition $\nu=$ $(r-2+p, r-3, r-4, \ldots, 1)$ obtained from $\mu$ by removing the rim $p$-hook and adding $p$ boxes to the first row. Then $\mu$ and $\nu$ belong to the same block since they have the same $p$-core and $|\mu|=|\nu|$, while $\nu$ belongs to the same block as $\kappa$ where $\kappa=(3 r-5, r-3, r-4, \ldots, 1)$ is obtained from $\nu$ by removing $p-2 r+3$ boxes in the first row. We conclude that $\lambda$ is in the same block as $\kappa$ and $|\kappa|=|\lambda|-2$. Furthermore, from Lemma 8.7 and the fact that $\nu$ is not a $p$-core, it follows that the 2-core of $\nu$ and thus also of $\kappa$ satisfies the conditions on $r$.

Hence for every $\lambda$ with $|\lambda| \geq 2$ there exists a $\mu$ such that $|\mu|=|\lambda|-2$ and $\lambda$ is contained in the same block as $\mu$ and for which the 2 -core of $\mu$ is an $r$-staircase which satisfies $2 r-1 \geq p$ or $\frac{r(r+1)}{2}+p-2 r \leq n$.

For each $\lambda \in \Lambda_{n}^{\prime}$, denote by $B_{n}(\lambda)$ the block algebra of $A_{n}$ containing the simple module $L_{n}(\lambda)$.

Theorem 8.11. The block decomposition of $A_{n}$ is given by

$$
B_{n}(\kappa) \oplus \bigoplus_{r} B_{n}\left(\rho_{r}\right)
$$

where the sum is over all $r \geq 2$ such that $2 r-1<p, \frac{r(r+1)}{2}+p-2 r>n$ and $\frac{r(r+1)}{2} \vdash n-2 k$ for some $k \geq 0$. Here $\rho_{r}$ is the $r$-staircase partition and $\kappa=(1)$ if $n$ is odd or $\kappa=(1,1)$ if $n$ is even.

In particular if $n \geq\left(p^{2}+7\right) / 8$, there is only one block.

Proof. The block decomposition follows immediately by combining Proposition 8.9 with Proposition 8.10 and noting that the partitions $\emptyset$ and $(1,1)$ are in the same block (since $W_{2}(\emptyset) \cong$ $\left.L_{2}(1,1)\right)$. Observe that if $n \geq\left(p^{2}+7\right) / 8$, then $2 r-1<p$ implies

$$
\frac{r(r+1)}{2}+p-2 r \leq \frac{(p-1)(p+1)}{8}+1=\frac{p^{2}+7}{8} \leq n,
$$

since $r(r+1) / 2-2 r$ is an increasing function of $r$ for $r \geq 2$. In particular there are no $r$ satisfying the condition of the summation, and so we only have one block.

\section{ACKNOWLEDGEMENT}

SB thanks Kevin Coulembier for helpful discussions and comments. SB is supported by a BOF Postdoctoral Fellowship from Ghent University.

\section{REFERENCES}

[BDEal18] M. Balagovi'c, Z. Daugherty, I. Entova-Aizenbud, I. Halacheva, J. Hennig, M. S. Im, G. Letzter, E. Norton, V. Serganova and C. Stroppel, The affine VW supercategory, https://arxiv.org/abs/1801.04178

[BSR98] G. Benkart, C. L. Shader, and A. Ram, Tensor product representations for orthosymplectic Lie superalgebras, J. Pure Appl. Algebra 130 (1998), no. 1, 1-48.

[BE17] J. Brundan and A. Ellis, Monoidal supercategories, Comm. Math. Phys. 351 (2017), no. 3, $1045-1089$. 
[CDM09a] A. G. Cox, M. De Visscher, and P. P. Martin, The blocks of the Brauer algebra in characteristic zero, Representation Theory 13 (2009), 272-308.

[CDM09b] A. G. Cox, M. De Visscher, and P. P. Martin, A geometric characterisation of the blocks of the Brauer algebra, J. London Math. Soc. 80 (2009), 471-494.

[Cou18] K. Coulembier, The periplectic Brauer algebra, Proc. Lond. Math. Soc. 117 (2018), no. 3, 441-482.

[CE18] K. Coulembier and M. Ehrig The periplectic Brauer algebra II: Decomposition multiplicities, J. Comb. Algebra, 2 (2018), no.1, 19-46.

[DWH99] W. F. Doran, D. B. Wales, and P. J. Hanlon, On the semisimplicity of the Brauer centralizer algebras, J. Algebra 211 (1999), 647-685.

[DR98] J. Du and H. Rui, Based algebras and standard bases for quasi-hereditary algebras, Trans. Amer. Math. Soc. 350 (1998), no. 8, 3207-3235.

[ES16] M. Ehrig and C. Stroppel, Schur-Weyl duality for the Brauer algebra and the ortho-symplectic Lie superalgebra, Math. Z. 284 (2016), no. 1-2, 595-613.

[GL96] J. J. Graham and G. I. Lehrer, Cellular algebras, Invent. Math. 123 (1996), 1-34.

[GM88] A. M. Garsia and T. J. McLarnan, Relations between Young's Natural and the Kazhdan-Lusztig Representations of $S_{n}$, Adv. Math. 69 (1988), 32-92.

[JK81] G. D. James and A. Kerber, The representation theory of the symmetric group, Encyclopoedia of Mathematics and its Applications 16, Cambridge University Press (1981)

[Kin14] O. King, The limiting blocks of the Brauer algebra in characteristic p, J. Algebra 397 (2014), 168-189.

[KT17] J. R. Kujawa and B. C. Tharp, The marked Brauer category, J. Lond. Math. Soc. (2) 95 (2017), no. 2, 393-413.

[KX01] S. König and C. C. Xi, A characteristic free approach to Brauer algebras, Trans. Amer. Math. Soc. 353 (2001), no. 4, 1489-1505.

[LZ17] G. I. Lehrer and R. B. Zhang, The first fundamental theorem of invariant theory for the orthosymplectic supergroup, Comm. Math. Phys. 349 (2017), no. 2, 661-702.

[Ma15] P. Martin. The decomposition matrices of the Brauer algebra over the complex field. Trans. Amer. Math. Soc. 367 (2015), no. 3, 1797-1825.

[Mat99] A. Mathas, Iwahori-Hecke algebras and Schur algebras of the symmetric group, University Lecture Series, 15. American Mathematical Society, Providence, RI, 1999.

[Moo03] D. Moon, Tensor product representations of the Lie superalgebra $\mathfrak{p}(n)$ and their centralizers, Comm. Algebra 31 (2003), 2095-2140.

[Mul79] G. Mullineux, Bijections on p-regular partitions and p-modular irreducibles of the symmetric groups, J. London Math. Soc. (2) 20 (1979), 60-66.

[Mur95] G.E. Murphy, The representation of the Hecke algebras of type $A_{n}$, J. Algebra 173 (1995), 97-121.

[Se14] V. Serganova, Finite dimensional representations of algebraic supergroups, Proceedings of the International Congress of Mathematicians, Seoul, 2014.

Email address: Sigiswald.Barbier@UGent.be

Department of Mathematical Analysis, Faculty of Engineering and Architecture, Ghent UniverSity, Krijgslaan 281, 9000 Gent, Belgium

Email address: A.G.Cox@city.ac.uk

Email address: Maud.Devisscher.1@city.ac.uk

Mathematics Department, City, University of London, Northampton Square, London, EC1V 0HB, ENGLAND 